\title{
Transfer Functions for Fields in a 3-mirror Nested Cavity
}

\author{
Malik Rakhmanov \\ LIGO Project, California Institute of Technology, Pasadena, CA 91125
}

\begin{abstract}
Transfer functions for the fields in a 3-mirror nested cavity are obtained. Explicit formulae for their poles and zeros are found. These results are used for simple analysis of the response of power recycling and signal recycling interferometers.
\end{abstract}

\section{INTRODUCTION}

Fabry-Perot cavities are an essential part of the optical topology of emerging interferometric gravitational wave detectors. It is well-known that the sensitivity of these detectors can be improved if one or two external cavities are formed by adding more mirrors to the interferometer. The result is equivalent to having a cavity inside a cavity or a nested cavity. Optical topologies with equivalent nested cavities are power recycling [1], signal recycling [2] and resonant sideband extraction [3]. In the first generation of the interferometric gravitational wave detectors the only equivalent nested cavity appears in the dynamics of the common mode of the arm-cavity motion and the laser frequency. The next generation of detectors may have two or more nested cavities. In this paper we obtain the transfer functions for fields in an arbitrary nested cavity and describe how to calculate the most important parameter of these transfer functions: the lowest order pole.

\section{FABRY-PEROT CAVITY}

We begin with a reminder of how the transfer function of a single Fabry-Perot cavity can be obtained. Let the length of the Fabry-Perot cavity be $L$ and the delay time $T(T=L / c)$. If the amplitude of the incident field is $E_{\text {in }}(t)$ then the amplitude of the field in the cavity, $E(t)$, is defined by the self-consistent equation

$$
E(t)=t_{a} E_{\text {in }}(t)+r_{a} r_{b} E(t-2 T)
$$

where $r_{a, b}$ is the reflectivity and $t_{a, b}$ is the transmissivity of the cavity mirrors. Figure 1 shows how the self-consistent field is formed.

CP523, Gravitational Waves: Third Edoardo Amaldi Conference, edited by S. Meshkov (C) 2000 American Institute of Physics 1-56396-944-0/00/\$17.00 
FIGURE 1. Self-consistent field in the Fabry-Perot cavity.

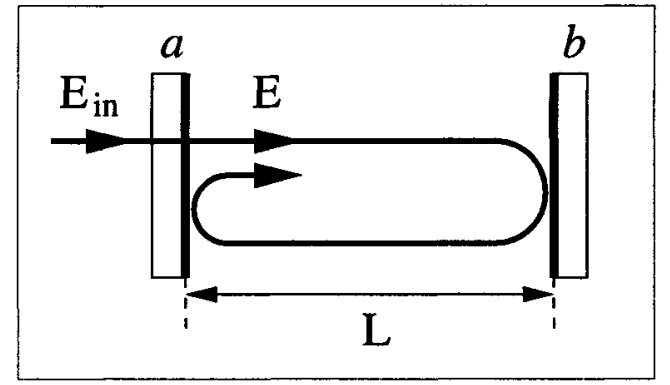

By applying the Laplace transformation to both sides of equation (1) we obtain the relation between the fields in $s$-domain:

$$
\tilde{E}(s)=t_{a} \tilde{E}_{\text {in }}(s)+r_{a} r_{b} \tilde{E}(s) e^{-2 s T}
$$

where tilde stands for the Laplace transform. Therefore, the cavity acts as a linear operator and its transfer function is

$$
H(s) \equiv \frac{\tilde{E}(s)}{\tilde{E}_{\text {in }}(s)}=\frac{t_{a}}{1-r_{a} r_{b} e^{-2 s T}} .
$$

This transfer function has an infinite set of poles:

$$
z_{n}=z_{0}+i \frac{\pi}{T} n, \quad \text { where } \quad z_{0}=\frac{\ln \left(r_{a} r_{b}\right)}{2 T}
$$

Here $z_{0}$ is the lowest order pole and $n$ is an integer.

\section{NESTED CAVITY}

A nested cavity can be formed by adding a mirror to the existing Fabry-Perot cavity, as shown in Figure 2. Then there will be two fields and their amplitudes, $E$ and $E^{\prime}$, satisfy the self-consistent equations:

$$
\begin{aligned}
E(t) & =t_{a} E^{\prime}\left(t-T^{\prime}\right)+r_{a} r_{b} E(t-2 T), \\
E^{\prime}(t) & =t_{c} E_{\mathrm{in}}(t)-\eta r_{a} r_{c} E^{\prime}\left(t-2 T^{\prime}\right)+\eta t_{a} r_{b} r_{c} E\left(t-T^{\prime}-2 T\right),
\end{aligned}
$$

where $\eta=e^{-2 i \phi}$ and $\phi$ is the detuning phase of the outer cavity. Let us apply the Laplace transformation to these equations. The result can be written as a matrix equation for complex 2-vectors:

$$
\mathbf{M}\left[\begin{array}{c}
\tilde{E} \\
\tilde{E}^{\prime}
\end{array}\right]=t_{c}\left[\begin{array}{c}
0 \\
\tilde{E}_{\mathrm{in}}
\end{array}\right],
$$


FIGURE 2. Self-consistent fields in a 3-mirror nested cavity with partial length $L$ and $L^{\prime}$.

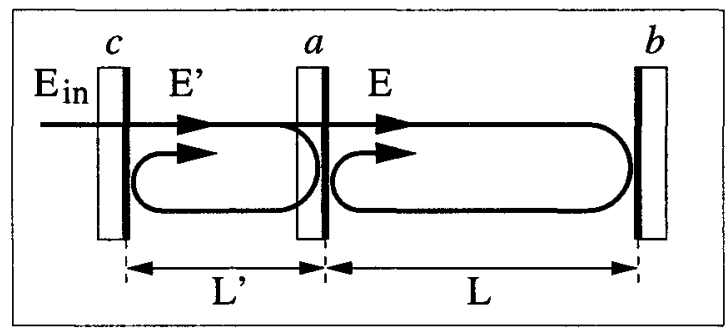

where $\mathbf{M}$ is the matrix function of $s$ :

$$
\mathbf{M}=\left[\begin{array}{cc}
1-r_{a} r_{b} e^{-2 s T} & -t_{a} e^{-s T^{\prime}} \\
-\eta t_{a} r_{b} r_{c} e^{-s T^{\prime}-2 s T} & 1+\eta r_{a} r_{c} e^{-2 s T^{\prime}}
\end{array}\right]
$$

By inverting the matrix we obtain the two transfer functions:

$$
\begin{aligned}
& G_{1}(s) \equiv \frac{\tilde{E}(s)}{\tilde{E}_{\mathrm{in}}(s)}=\frac{-t_{c} M_{12}}{\operatorname{det} \mathbf{M}}, \\
& G_{2}(s) \equiv \frac{\tilde{E}^{\prime}(s)}{\tilde{E}_{\mathrm{in}}(s)}=\frac{t_{c} M_{11}}{\operatorname{det} \mathbf{M}}
\end{aligned}
$$

These transfer functions share the same poles, which are defined by

$$
\operatorname{det} \mathbf{M} \equiv 1-r_{a} r_{b} e^{-2 s T}+\eta r_{a} r_{c} e^{-2 s T^{\prime}}-\eta\left(t_{a}^{2}+r_{a}^{2}\right) r_{b} r_{c} e^{-2 s\left(T^{\prime}+T\right)}=0 .
$$

In addition, the transfer function $G_{2}(s)$ has zeros, which are defined by $M_{11}=0$. Note that the zeros are $s=z_{n}$, where $z_{n}$ are given by the equation (4). In other words, the zeros of the outer cavity appear at the frequencies where the poles of the inner cavity were before the nested cavity was formed. This is a general result.

The equation for the poles (eq. (11)), cannot be solved analytically, unless some approximations are made. In the low frequency approximation we replace the exponents by linear functions and obtain the solution for the lowest order pole:

$$
s_{0} \approx \frac{1+\eta r_{a} r_{c}-r_{a} r_{b}-\eta\left(t_{a}^{2}+r_{a}^{2}\right) r_{b} r_{c}}{2\left[\eta r_{a} r_{c} T^{\prime}-r_{a} r_{b} T-\eta\left(t_{a}^{2}+r_{a}^{2}\right) r_{b} r_{c}\left(T^{\prime}+T\right)\right]}
$$

This approximation is valid as long as $\left|s_{0}\right| T \ll \pi$, that is the frequency of the pole must be much less than the free spectral range of the longer cavity.

Another formula for the lowest order pole can be obtained if one of the cavities is much shorter than the other. Assume that $T^{\prime} \ll T$ then we can neglect $T^{\prime}$ in equation (11) and obtain the solution

$$
s_{0} \approx \frac{1}{2 T} \ln \left[\frac{1+\eta r_{a} r_{c}}{r_{a} r_{b}+\eta\left(t_{a}^{2}+r_{a}^{2}\right) r_{b} r_{c}}\right],
$$


which is valid as long as $\left|s_{0}\right| T^{\prime} \ll \pi$, that is the frequency of the pole must be much less than the free spectral range of the shorter cavity.

At low frequencies the transfer functions can be approximated up to a constant by a low-pass and a lead-lag filter respectively:

$$
G_{1}(s) \approx \frac{1}{s-s_{0}}, \quad G_{2}(s) \approx \frac{s-z_{0}}{s-s_{0}} .
$$

The formulae above can be used for a simple analysis of optical response of gravitational wave detectors. First consider a power recycling interferometer, for example, the $4 \mathrm{~km}$ LIGO interferometer. Its equivalent nested cavity appears in the dynamics of the common mode of the arm motion. The target bandwidth of the detector sets the lowest order pole of the arm cavity: $\left|z_{0}\right| / 2 \pi=90 \mathrm{~Hz}$. This condition defines the transmission of the input mirror $\left(t_{a}^{2}=0.03\right)$ provided the transmission of the end mirror is fixed $\left(t_{b}^{2}=20 \mathrm{ppm}\right.$.) The condition for optimal coupling of the laser power to the interferometer defines the transmission of the recycling mirror $\left(t_{c}^{2} \approx 0.024\right)$. The result of the recycling is that the circulating power reaches $16 \mathrm{~kW}$ for an input power of only $6 \mathrm{~W}$. From either equation (12) or (13) with $\eta=1$ we find that the lowest order pole for the common mode is $\left|s_{0}\right| / 2 \pi=1.1 \mathrm{~Hz}$. Thus the transfer function of the power recycling cavity has a pole at $1.1 \mathrm{~Hz}$ and a zero at $90 \mathrm{~Hz}$.

Now consider an interferometer with LIGO parameters, which has a signal recycling mirror instead of a power recycling mirror. Then an equivalent nested cavity appears in the dynamics of the differential mode of the arm motion. Such an interferometer can be analyzed as follows. The optimal coupling of the laser power to the arm cavities defines the transmission of the input mirror $t_{a}^{2}=120 \mathrm{ppm}$ under the assumption that the losses are $50 \mathrm{ppm}$ per mirror. This sets the arm cavity pole $\left|z_{0}\right| / 2 \pi=0.7 \mathrm{~Hz}$, and also results in a high circulating power (25 $\mathrm{kW}$ for the same $6 \mathrm{~W}$ of input power). The reflectivity of the signal recycling mirror will define the detection bandwidth according to the equation (12) or (13) with $\eta=-1$. These equations show that in order to obtain the same $90 \mathrm{~Hz}$-bandwidth the signal recycling mirror must have transmission $t_{c}^{2}=0.011$. Note that the transfer function of the signal recycling cavity will have a pole at $90 \mathrm{~Hz}$ and a zero at $0.7 \mathrm{~Hz}$. This means that in general the response of the cavity will be poor at low frequencies, and, therefore, it will be hard to control the signal recycling mirror. These two examples show how the formulae for the lowest order pole of a nested cavity can be used in a simple analysis of the interferometer response.

\section{REFERENCES}

1. Drever, R.W.P, in Gravitational Radiation, eds. N. Deruelle and T. Piran, North Holland, Amsterdam, 1983.

2. Meers B.J., Phys. Lett. A142, 465, (1989).

3. Mizuno, J., Comparison of optical configurations for laser-interferometric gravitational-wave detectors, $M P Q-$ Report 203, (1995). 